\title{
Generation System for Renewable Energy Density Substantially Higher than that of Power Stations and Presentation of Novel Superconductivity
}

\author{
Shinichi Ishiguri \\ Nihon University \\ 1-2-1 Izumi-Cho, Narashinoshi, Chiba 275-8575 JAPAN \\ TEL: +81-47-474-9143 \\ Email: ishiguri.shinichi@nihon-u.ac.jp
}

\begin{abstract}
Societies around the world face serious energy problems related to the consumption of fossil fuels and the emission of dangerous radiation. To solve these problems, a new superconductor exhibiting a critical temperature higher than room temperature has been pursued but not achieved. This paper proposes a new energy generation system based on a circuit approach. Secondary to this process, a new type of superconductivity without refrigeration is demonstrated. In our previous paper [1], this system was proposed, but it did not describe the underlying theory in detail and did not mention an actual method to generate energy from the system. The present paper describes the theory of the existence of divergent current density and new superconductivity with no refrigeration. Moreover, the present paper proposes a method for extracting energy from the system by employing a voltagecontrolled current source (i.e., a voltage-current converting method).

The principle of the system is based on a circuit of two loops and independent current sources. First, the two electric loops are prepared, each with 4 diodes, where the diodes are oriented in the same direction within each loop, but their direction is opposite from loop to loop; four independent current sources connect the loops. In this circuit system, current is added iteratively as it flows along the loop according to Kirchhoff's circuit law. As a result, a large current and electric potential are present along the loop. To confirm that this system works properly, it is necessary to demonstrate the presence of divergent currents in the transient state, and to do this, the present paper employs the Dirac equation and Lorentz conservation. Electric circuit software is employed to demonstrate that the presented method generates energy actually from our system.

Our results confirm the presence of divergent current at a connected point of an independent current source in the transient state. Moreover, in the steady state, the theory demonstrates the Meissner effect (i.e., a London equation) and a new type of macroscopic wave function and condensation. For an initial small input current of $0.1 \mu \mathrm{A}$, the simulation reveals a large generating current of $7 \mathrm{kA}$ and electric power of $10^{11} \mathrm{~W}$, which is much larger than unit of power from an average thermal power station; moreover, the system presents with superconducting electrical transport conditions.
\end{abstract}


The present study is significant because it demonstrates theoretically the existence of divergent current density and a new type of superconductivity requiring no refrigeration. Secondly, the simulations show the generation of a large energy density that can be obtained in a small laboratory room with minimal cost.

\section{Keyword}

Divergent current density, New superconductivity, Meissner effect, New type of condensation, Energy generation, Electrostatic potential, Independent current source, Voltage-controlled current source, Renewable energy

\section{Introduction}

This paper discusses a new systematic renewable energy source that produces electric power larger than the unit of power generated from an average thermal power station. The system can be set up in a small laboratory without consuming fossil fuels or emitting radiation. Moreover, the existence of divergent current density and a new type superconductivity without refrigeration are demonstrated in both theory and simulations.

To understand the significance of this study, it is helpful to be aware of the background, i.e., the general situation facing global society. As is becoming increasingly clear, our earth is facing serious energy problems, which is in part the result of high fossil fuel consumption. In addition to increasing concern that finite fuels will be depleted in the near future, fossil fuel consumption, which is a common driver of thermal power stations, has led to an increase in $\mathrm{CO}_{2}$ concentration in the atmosphere. In addition, thermal power stations still have low efficiency in terms of energy conversion, which results in further increase in $\mathrm{CO}_{2}$ concentration. Furthermore, there are serious problems regarding the use of nuclear power stations. Japan experienced a huge earthquake in 2011 that was estimated to be the strongest of the past 1000 years. The nuclear power station in the Fukushima Prefecture fell victim to the subsequent tsunami; its sustained damage and destruction resulted in significant emission of dangerous radiation such as Ce isotopes. The Fukushima nuclear power station has not been restored, and the people in the surrounding area still suffer from losing their homes.

Many other countries including Japan are trying to promote the use of renewable energy such as solar energy, wind energy [2] and so on. However, a crucial disadvantage of the current state of available renewable energy technologies is that they generate a low amount of energy despite large investments in plants and equipment. Although the efficiencies of existing renewable energy have increased greatly, it is estimated that every existing renewable energy will only be able to generate $30 \%$ of the energy required to meet the demand [3]. Consequently, it is likely that society will be dependent on thermal and nuclear power stations for most of the energy demands.

To meet the global energy demands, new energy generating principles must be created that enable 
the generation of electric power much larger than that of thermal and nuclear power stations. The current paper is directly related to our previous report on a new principle to generate energy [1]. In our proposed system, first an electric circuit loop including 4 diodes is prepared whose directions are all the same. Then, another loop is prepared with 4 diodes whose directions are also same with respect to each other; however, the direction of the prepared diodes is opposite from loop to loop. Four independent current sources connect the prepared two loops. When applying Kirchhoff's current law along a loop, an independent current source first adds a current to the loop; then, another current source adds an additional current. The 4 current sources iteratively add current, but the added current does not leave the loop (i.e., only current additions occur along a loop). As described in the theory section (see Section 3) in the present paper, it is implied that a divergent current is present in the transient state, resulting in large currents and electric potentials on the loops; this was also demonstrated in our previous paper [1]. Moreover, our previous work revealed that no voltage appeared along a loop in the steady state, which agrees with Kirchhoff's voltage law and satisfies a condition for generating superconductivity [1].

However, our previous paper did not describe the underlying theories in detail and did not propose a concrete method to actually generate energy from the large electric potentials. When compared with our previous report, the present work features progress as summarized in the following points:

[1] Theory describing the phenomenon is presented. Here, by using Lorentz conservation, the Dirac equation provides divergent current density in a transient state, whereas the steady state gives rise to a London equation (i.e., the Meissner effect), a macroscopic wave function, and a new type of condensation.

[2] Method to generate energy from this system is presented and numerical simulations demonstrate its effect. Considering that a loop has a uniform and large electric potential (i.e., there is no voltage along the loop), the goal was to develop a method to generate energy from the system. In the present paper, a voltage-controlled current source is proposed. In comparison to an independent current source, this dependent current source works to precisely convert an input voltage to an output current. Therefore, the device does not generate energy. Of the two loops in our system, one undergoes a positive and uniform electric potential, and the other takes a negative and uniform electric potential. It follows that among the input taps of the voltage-controlled current source, one tap receives a positive electric potential from one loop, and the other tap receives a negative electric potential from the other loop. In this way, a large direct current (DC) voltage can be input to the voltage-controlled current source. By employing Powersim PSIM electric circuit simulation software, we find that for an initial input current of $0.1 \mu \mathrm{A}$ from an independent current source, the resulting output current from the voltage-controlled current source becomes approximately $7 \mathrm{kA}$; therefore, employing a sufficiently large load provides much larger generated electric power than a unit of power from an average thermal power station. Furthermore, this energy generation system can be set up in an average and small 
laboratory room with at a low cost estimated to 100,000 yen $(<1,000$ USD). Moreover, it generates no radiation and consumes no fossil fuels.

On the other hand, the present paper signifies progress in condensed matter physics, i.e. our proposed system exhibits a new type of superconductivity without refrigeration. The first superconductor, $\mathrm{Hg}$, was found nearly a century ago and required significant refrigeration to approximately $4 \mathrm{~K}$. Generally, a superconductor is believed to become superconducting when cooled below its critical temperature. Therefore, a higher critical temperature is preferred in view of operation costs. If a superconductor exhibits a critical temperature higher than room temperature, many applications will be enabled that could eventually lead to a profound breadth of applications including those that may solve the aforementioned global energy problems. Since discovering the superconducting properties of $\mathrm{Hg}$, extensive research has been carried out in the search for new superconductors possessing higher critical temperatures [4-8]. Over the years, some remarkable information and materials were uncovered such as $\mathrm{CuO}_{2}$-based high-temperature superconductors whose critical temperatures are higher than that of $\mathrm{LN}_{2}$ [9], $\mathrm{MgB}_{2}$ metal-based superconductivity [10], Fe-based superconductors [11] and so on. Moreover, in condensed matter physics, Bardeen-CooperSchrieffer theory was developed [12], which describes the mechanism of metal superconductors, and the quantum tunneling of Cooper pairs, the Josephson Effect [13], was observed.

In short, the common goal of superconductivity research is to find a superconductor with a critical temperature higher than room temperature and requiring no pressure; such an achievement would also present a solution to the global energy problem and promote quantum computing. We would like to note that recently work has shown that some materials are superconducting near room temperature when subjected to extremely high pressure (250 GPa) [14]. Although these superconductors are significant in condensed matter physics, it is difficult to use them in device applications due to the extremely high pressure requirement. In summary, despite thorough experimental and theoretical research, no room-temperature superconductor operating under normal atmospheric pressure has been reported

In the present paper, a new type of superconductivity is demonstrated in both theory and simulations; it emerged during the development of the aforementioned energy generating system. This uncovered superconductivity is generated with no refrigeration because critical temperature, a superconducting energy gap, and the combination energy among two electrons do not play a role in this system. We present a London equation (i.e., the Meissner effect) and a macroscopic wave function with particle condensation as well as demonstrate in simulations that there is of no voltage generation. We have already published similar reports regarding generating superconductivity without refrigeration [15-18]. However, those reports are based on the combination energy of two electrons and a superconducting energy gap, which are much higher than room temperature energy. Therefore, the setup and results presented in this paper are independent of the idea of a critical temperature. In 
addition to the contributions provided in this paper that were reviewed in the aforementioned items [1] and [2], this paper also describes a new kind of superconductivity.

[3] In the steady sate, the present paper describes a new type of superconductivity requiring no refrigeration both theoretically and by using simulations. In particular, this paper demonstrates a new concept of fermion condensation that does not contradict the Pauli exclusion principle.

The contributions of this paper are as follows:

(1) Our work represents a significant theoretical contribution because our results demonstrate the existence of divergent current density and temperature-independent superconductivity. As far as we know, we are the first to report this.

(2) By employing our proposed system, high energy density can be obtained that is much larger than that of average thermal power stations; it consumes no fossil fuels and emits no radiation. Furthermore, this system does not require investment in an expensive plant or special equipment. As will be described in Discussion section, the energy loss can be considered nearly zero when compared with the high output energy.

\section{Principle}

2.1 Review of an Independent Voltage Source, an Independent Current Source, and a VoltageControlled Current Source

Generally, power supplies are divided into two typical types: independent voltage and current sources. As shown in Fig. 1-[a] and [b], an independent voltage source provides a constant voltage to a connected load regardless of the load, and thus a current passing the voltage source is varied according to Ohm's law. Moreover, its internal resistance is ideally zero.

On the other hand, an independent current source provides a constant current independent of the load; hence, a voltage drop between the taps of the current source is determined by Ohm's law. Moreover, its internal resistance is ideally infinite, which is an important fact in our system.

A voltage-controlled current source is a type of voltage to current converter. Therefore, a voltage-controlled current source does not generate energy. It is employed because of the large resulting electric potential, which is uniform along a loop, i.e., it is necessary to create a method to achieve energy gain from the uniform electrostatic potentials because there is no voltage. However, the two loops in our system have opposing (signs + or - ) electric potentials. By taking advantage of this fact, we can harvest energy by applying a voltage-controlled current source; a circuit diagram of a voltage-controlled current source is shown in Fig. 1-[c]. A potential configuration of this device is 
employing an operational amplifier (op-amp) since one implementation of an op-amp is to convert a voltage to a current. Among various integrated circuits (ICs), the ICLM741 is a candidate for this setup. This type of IC provides an accurate current upon applying an input voltage.

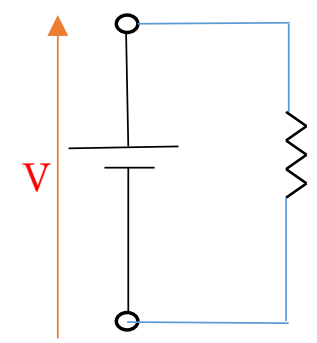

[a]

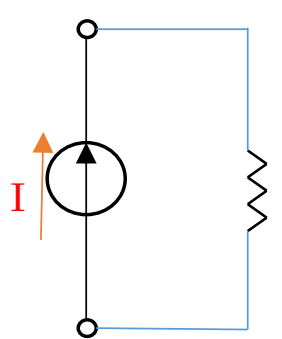

[b]

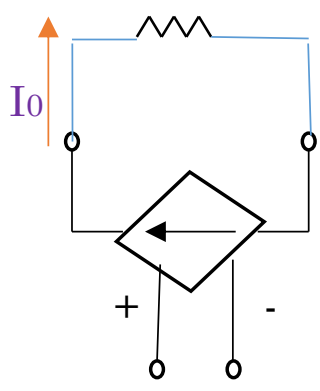

Vin

[c]

Fig.1

Schematic diagrams of an independent voltage source [a], an independent current source [b], and a voltagecontrolled current source [c]. An independent voltage source provides a constant voltage to the load regardless of the load. Therefore, its current varies on the load according to Ohm's law. An independent current source supplies a constant current regardless of the load; hence, the voltage drop between the taps of the current source varies based on the load. A voltage-controlled current source works to convert a voltage to a current with precise amount. It does not generate energy by itself.

We consider the configuration of a voltage-controlled current source. In Fig. 2, the structure of the device is shown. The non-inverting terminal of an op-amp is connected to input voltage $V_{\text {in }}$, and the inverting terminal is connected to internal conductance $\mathrm{k}$. Moreover, a load $\mathrm{R}_{\mathrm{L}}$ is floating (i.e., not grounded). The inverting terminal is fed by the feedback voltage that arises due to the load $\mathrm{R}_{\mathrm{L}}$ and the voltage $V_{D}$ of the op-amp. 


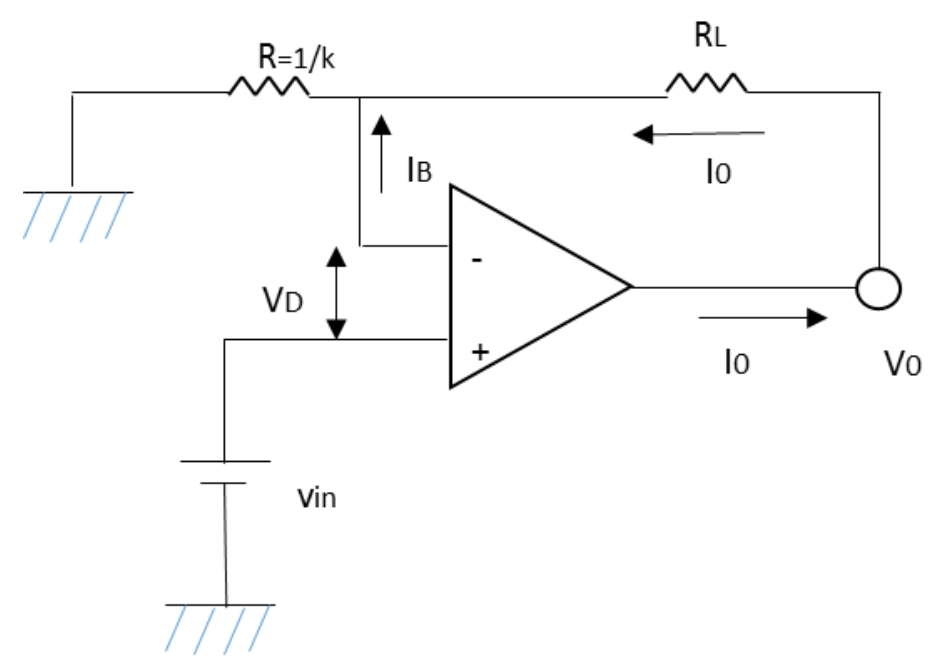

Fig 2.

Internal configuration of a voltage-controlled current source. This device is a voltage-current converter. Due to the imaginary short of an op-amp, $V_{D}$ and $I_{B}$ are approximately zero, hence the output current $I_{0}$ passing the load $R_{L}$ is determined by the internal conductance $k$ (i.e., $R=1 / k$ ), which is set to 1 in this paper.

Considering Kirchhoff's voltage law along the input loop,

$$
V_{\text {in }}=V_{D}+V_{F} \text {. }
$$

Because of the imaginary short of an op-amp, which is a unique characteristic of every op-amp and the result of the extremely large gain in the op-amp,

$V_{D} \approx 0$.

Therefore,

$V_{\text {in }}=V_{F}$.

The current $\mathrm{I}_{\mathrm{B}}$ indicated in Fig. 2 is approximated as zero; hence, the configuration can be described by the following equation,

$$
I_{0}=k V_{\text {in }}
$$

where the output current $\mathrm{I}_{0}$ flows through the internal conductance $\mathrm{k}$ as well as the load $\mathrm{R}_{\mathrm{L}}$. Equation (Eq. 4) implies that the output current $\mathrm{I}_{0}$ is controlled by the internal conductance $\mathrm{k}$; here, it is set to 1 . In this way, the input voltage $\mathrm{V}_{\text {in }}$ is precisely converted to the output current $\mathrm{I}_{0}$. 


\subsection{Review of our Previous Paper}

Figure 3 presents two electrical loops that each have 4 diodes (note that this figure is taken from our previous paper [1]). The diodes are oriented in opposite directions from loop to loop, i.e., one loop has the diodes pointing clockwise, and the diodes in the other loop are oriented in a counter-clockwise arrangement. Then, as shown in Fig. 4, four current sources are connected between the loops.

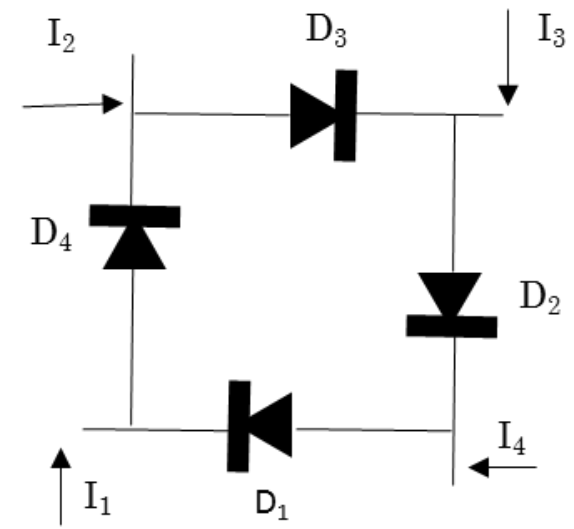

(a)

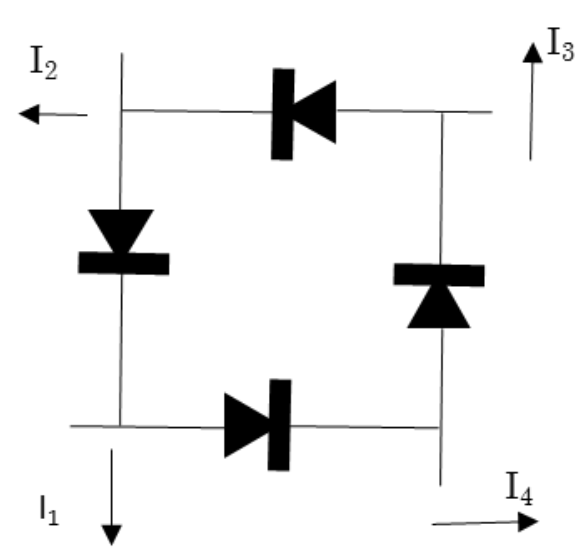

(b)

Fig. 3

Schematic of the principle featured in the proposed system. Note that this figure was originally published in our previous our paper [1]. The system is composed of two electric loops. In each loop, the four diodes are placed in the same orientation. However, when connecting the two loops, the diode directions per loop are opposite. The loops are connected by 4 independent current sources. For example, currents $I_{1}$ in (a) and (b) are connected with an independent current source. The current input along the loop in (a) builds iteratively around the loop, resulting in a large output current and a large electric potential.

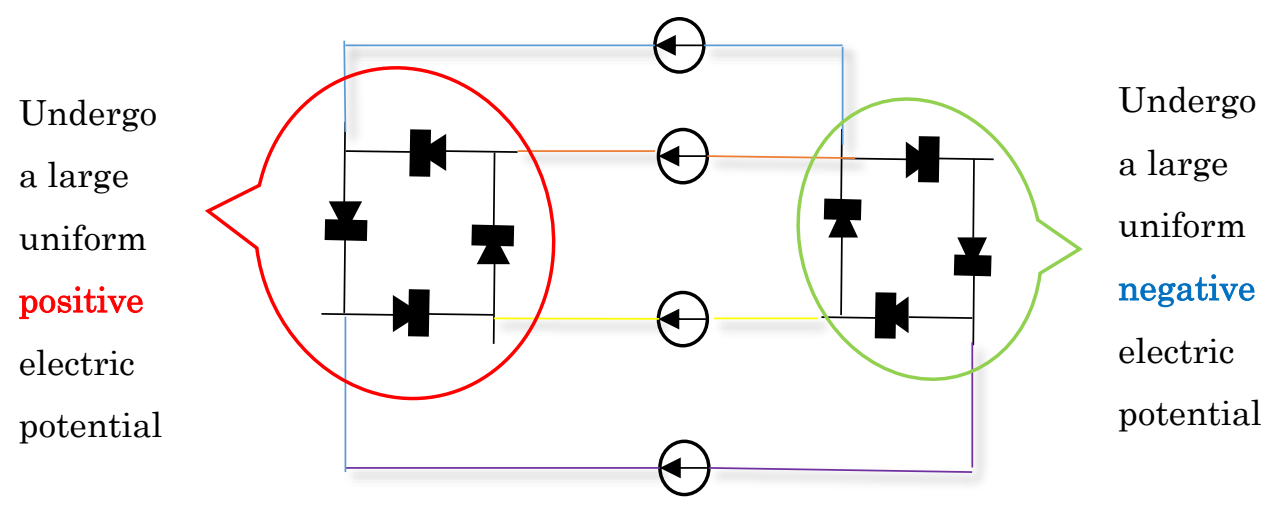

Fig. 4

Overview of the proposed system. As described in Fig. 3, two loops containing diodes are prepared and connected 
by 4 independent current sources.

As will be discussed in the Theory section and is presented in Fig. 3, the connecting point from a current source generates divergent currents, i.e., Kirchhoff's current law results in iterative addition of current. For example, consider the left loop in Fig. 3. First, current $I_{1}$ is input to the loop, and it passes diode $D_{4}$. Then, current $I_{2}$ is added to current $I_{1}$, and the increased current passes diode $D_{3}$. Next, $I_{3}$ is added to $I_{1}$ and $I_{2}$. These increased currents pass diode $D_{2}$, and then current $I_{4}$ is added. The next step is the same, i.e., the iterative current addition occurs. As a result, a substantially large current is generated. Moreover, a large electric potential is exhibited along the loop.

The above described phenomenon is in the transient state; at steady state, according to Kirchhoff's voltage law, the total voltage is zero along a loop. Therefore, we can predict that superconductivity appears. As will be discussed in Theory section, a London equation (i.e., the Meissner effect) is obtained. Because the currents are added many times in the transient state, a very large electric potential and superconducting current are generated uniformly along a loop. Note that the electric potential at one loop is positive while the other loop simultaneously has a negative electric potential. As mentioned, this fact is important when energy gain is desired via the application of a voltage-controlled current source.

\subsection{How to Generate Energy from the System}

In order to generate net power from the system, it is useful to use the substantially large electric potentials along the electric circuit, which are a result of our theory and simulations in our previous and current papers. As presented in Figs. 3 and 4, there are two electric loops connected by 4 independent current sources. The signs of the electric potentials of the loops are opposite, based on which a voltage-controlled current source is used to extract energy from the system, as presented in Fig. 5. 


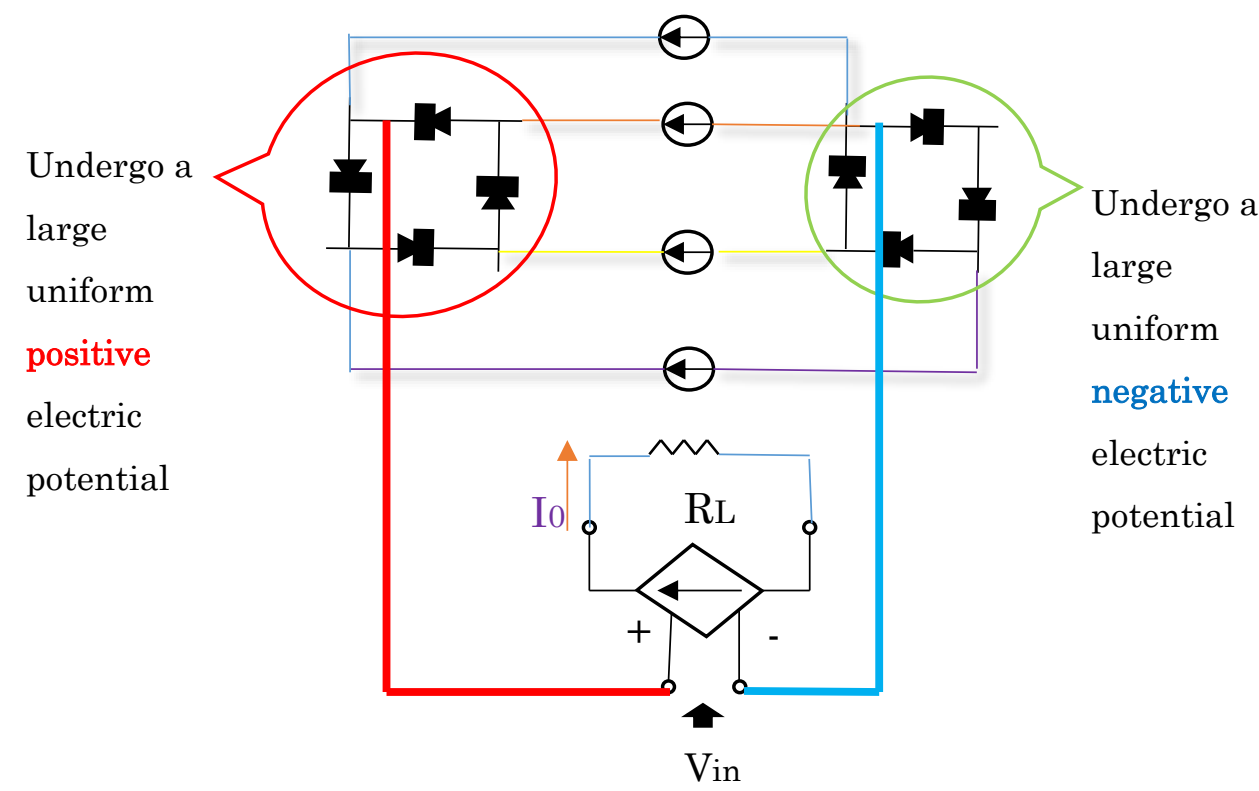

Fig.5

Schematic diagram showing the proposed method for extracting energy from the system. In our previous paper, it was clarified that iterations of current input result in large electric potentials. However, the electric potential is distributed uniformly along a loop and hence there is no net voltage.

In the present paper, one loop undergoes a large positive electric potential, and the other undergoes a large negative electric potential. When employing a voltage-controlled current source, one tap of the input source is connected to the loop with a positive electric potential, and the other tap of the input of the source is connected to other loop having a negative electric potential. In this way, a large input voltage $V_{\text {in }}$ is introduced via the voltage-controlled current source.

As shown in Fig. 5, among the two input taps of a voltage-controlled current source, one tap is connected to the loop with a positive electric potential, and the other tap is connected to the other loop having a negative electric potential, hence a large net voltage $\mathrm{V}_{\text {in }}$ (i.e., a creating a net voltage between the two electric potentials) is generated.

Because the 2 input taps of the voltage-controlled current source are independent of each other in terms of current, almost zero current flows into the input taps of the voltage-controlled current source. The input voltage stems from electrostatic fields, not Ohmic electric fields; therefore, the presence of large input currents to the voltage-controlled current source can be ignored. Such currents are determined by Ohmic electric fields, not electrostatic fields, according to Maxwell's equations. When a large voltage on the order of $\mathrm{kV}$ is the input, therefore, the input impedance of the voltagecontrolled current source is not broken. Therefore, only the loss Joule heating generates (see Eq. [4]) 
is considered. That is, the resistance $1 / \mathrm{k}(=1 \Omega)$ generates the Joule heating. As described in Discussion section, to reduce this heating, a DC-DC converted method will be applied to the input taps of the voltage-controlled current source.

\section{Theory}

\subsection{Lorentz Conservation}

In the proposed system, the following two Lorentz conservations hold:

$A_{t}^{2}-\left(\frac{\phi_{t}}{c}\right)^{2}=\left(\frac{1}{c e} \frac{1}{2} \hbar \omega\right)^{2}$

$\left(\hbar k_{t}\right)^{2}-\left(\frac{\varepsilon_{t}}{c}\right)^{2}=\left(\hbar k_{i n}\right)^{2}$.

Equation (5) is true in the transient state, where $A_{t}, \phi_{t}, e, c$, and $\omega$ denote the vector potential, electrostatic potential, charge of an electron, speed of light, and angular frequency, respectively. Similarly, Eq. (6) describes the transient state, where $\mathrm{k}_{\mathrm{t}}, \varepsilon_{\mathrm{t}}$, and $\mathrm{k}_{\text {in }}$ denote wavenumber, energy, and the wavenumber related to the input current from an independent current source.

The relationship between Eqs. (5) and (6), i.e., the energy conservation, is assumed to be given by

$\frac{1}{2} \hbar \omega=\frac{\hbar^{2} k_{i n}^{2}}{2 m}$

where $\mathrm{m}$ denotes the mass of an electron.

The zero-point energy $\frac{1}{2} \hbar \omega$ (as a part of the right-hand side of Eq. (5)) and the wavenumber $k_{\text {in }}$ represent the energy from the input-current source and the carrier momentum related indirectly to the input-current, respectively.

Equations (5) and (6) describe the transient state, but the steady state requires that the right-hand sides are consumed, i.e., the zero-point energy is consumed, (see Eqs. [5-2] and [6-2]). This results in a larger vector and an electrostatic potential, thus implying that the state transfers from the transient state to the steady state;

$A^{2}-\left(\frac{\phi}{c}\right)^{2}=0$,

$(\hbar k)^{2}-\left[\frac{1}{c}(-e \phi)\right]^{2}=0$

where

$\mathrm{A} \gg A_{t}$,

$\phi \gg \phi_{t}$, 
$\mathrm{k} \gg k_{t}$,

and

$\mathrm{e} \phi \gg \varepsilon_{t}$.

As mentioned, considering these conditions (Eqs. 7, 8, 7-2, and 8-2), the vector potential and the electrostatic potential become much larger than their initial values.

\subsection{Dirac Wave Function}

When up- and down-spin electrons are considered, the zero-point energy as a part of the right-hand side of Eq. (5) becomes

$2 \times \frac{1}{2} \hbar \omega=\hbar \omega=n \hbar \omega_{0}$,

where $\mathrm{n}$ is an integer that increases with input-current density from an independent current source, and $\omega_{0}$ is constant defined by

$\hbar \omega_{0}= \pm \sqrt{\left(m c^{2}\right)^{2}+c^{2}\left(\hbar k_{\alpha}\right)^{2}}$.

Eq. (9-2) represents the eigenvalue of the Dirac equation where up- and down-spin electrons are considered. Note that this paper only considers the positive eigenvalue in Eq. (9-2). Here, because we consider both up- and down-spin electrons, the wave function in the transient state becomes

$\psi_{0}=\psi_{\uparrow}+\psi_{\downarrow} \rightarrow \exp \left[\mathrm{j}\left(k_{\alpha} z-\omega_{0} t\right)\right]\left[\begin{array}{l}\cos \theta \\ \cos \theta\end{array}\right] \rightarrow 2 \exp \left[\mathrm{j}\left(k_{\alpha} z-\omega_{0} t\right)\right] \cos \theta$

where $\mathrm{j}$ is the imaginary unit.

In Eq. (10), $\theta$ is given by

$\tan 2 \theta=\frac{\hbar k_{\alpha}}{m c}$.

Considering the schematic diagram of the proposed principle (i.e., Figs. 3 and 4), we consider only a connecting point from an independent current source. That is, the $\mathrm{z}=0$ is substituted in the wave function.

$\psi=2 \exp \left(-j \omega_{0} t\right) \cos \theta$.

\subsection{Normalization of the Dirac Wave Function}

First, in the previous mentioned Dirac wave function (12), the normalization by volume integral is considered:

$\int|\psi|^{2} d v=1$.

Considering Eq. (13) and that the wave function only plays a role at the connecting point, the following equations should hold:

$\cos \theta=\frac{1}{2}$ 
$\theta=\frac{\pi}{3}+2 n \pi$

Furthermore, the wave function must follow:

$\psi \rightarrow \psi_{D} \equiv\{\delta(z)\}^{3 / 2} \exp \left(-j \omega_{0} t\right)$.

Note that this implies that the wave number $\mathrm{k}_{\alpha}$ defined by the Dirac equation is constant.

This paper requires the normalization of the time integral as well as that of the volume integral.

$\psi_{D} \rightarrow \psi_{e} \equiv\left|\psi_{t}\right|\{\delta(z)\}^{3 / 2} \exp \left(-j \omega_{0} t\right)$

Therefore, the general normalization is defined as

$\int\left|\psi_{e}\right|^{2} d v d t=1$.

Considering the characteristic of the Dirac delta function $\delta(z)$, Eq. (17-1) becomes

$\int_{0}^{T}\left|\psi_{t}\right|^{2} d t=1$,

where $\mathrm{T}$ denotes the period for angular frequency $\omega_{0}$.

As a result,

$\left|\psi_{t}\right|^{2}=\frac{\omega_{0}}{2 \pi}$.

In general, therefore, the resulting wave function is

$\psi_{e}=\sqrt{\frac{\omega_{0}}{2 \pi}}\{\delta(z)\}^{3 / 2} \exp \left(-j \omega_{0} t\right)$

Considering the dimension of the Dirac delta function $\delta(z)$,

$\left|\psi_{e}\right|^{2}=\frac{\omega_{0}}{2 \pi}\{\delta(z)\}^{3} \equiv \frac{1}{e} \frac{\partial|\rho|}{\partial t}$,

where $\rho$ denotes the charge density, which correlates to a negative charged electron.

Next let us consider the equation of continuity,

$-\frac{\partial|\rho|}{\partial t}+\operatorname{div} \vec{\jmath}=0$

Substituting with Eq. (20), the following result is obtained:

$\operatorname{div} \vec{\jmath}=\frac{e \omega_{0}}{2 \pi}\{\delta(z)\}^{3} \neq 0$.

Based on the divergence theorem, at $\mathrm{z}=0$ (i.e., at a connecting point from the independent current source), a divergent current from the current source appears. In the transient state, therefore, it is true that a current from the current source is input to the connecting point. It is thus confirmed that the proposed system is theoretically sound.

\subsection{Superconductivity at the Steady State}

The previous calculations were conducted for the transient state. Now we consider the steady state. In the steady state, the following equations hold:

$A^{2}-\left(\frac{\phi}{c}\right)^{2}=0$ 
$(\hbar k)^{2}-\left[\frac{1}{c}(-e \phi)\right]^{2}=0$

$\psi_{s}=\left|\psi_{s}\right| \exp (j k x)$,

$\int\left|\psi_{s}\right|^{2} d v=1$

Equations (23) and (24) imply that in the general Lorentz conservations, the right-hand sides were consumed, resulting in making the vector potential $\mathrm{A}$, electrostatic potential $\phi$, and wave number $\mathrm{k}$ much larger.

Moreover, Eq. (25) suggests a plane-wave whose wave number is determined by eq. (24). Furthermore, Eq. (25-2) defines Eq. (25) as a wave function.

Based on Eqs. (23) and (24), substituting the electric potential $\phi$ produces the following equation:

$\mathrm{A}=-\hbar \mathrm{k} \frac{c}{e}$.

Employing the probability flux equation, Eq. (27), results in a London equation (Eq. 28).

$j_{q}=e\left|\psi_{s}\right|^{2} \frac{\hbar k}{m}$,

$j_{q}=-\frac{e^{2}\left|\psi_{s}\right|^{2}}{m c} A$

Therefore, we can conclude that the Meissner effect occurs in the proposed system.

Next we consider whether a macroscopic wave function is formed.

As discussed, each electron wave function is represented as

$\psi_{s}=\left|\psi_{s}\right| \exp \left(-\frac{e \phi}{\hbar c} j x\right)$,

where

$|k| \equiv \frac{e \phi}{\hbar c}$.

Note that the wave function only holds along one dimension. It is important to note that generally every physics textbook describes wave numbers in a metal as a function of kinetic energy, which must follow Fermi statistics (i.e., the exclusion principle). However, here, the wave number is a function of the electrostatic potential $\phi$, which allows each electron to have an identical wave number. Therefore, as shown in Fig. 7, each wave number $\mathrm{k}$ can be summed as scalars; although each wave function is allowed to exist at each point, only wave numbers may be added. It is possible to consider that a total wave number $\mathrm{K}$ along the circuit can be expressed by Eq. (30) without contradicting the exclusion principle:

$\mathrm{K}=\sum k=N \frac{e \phi}{c \hbar}$,

where $\mathrm{N}$ is an integer. Therefore, the electric potential becomes large: 
$\mathrm{N} \phi \equiv \Phi_{T}$

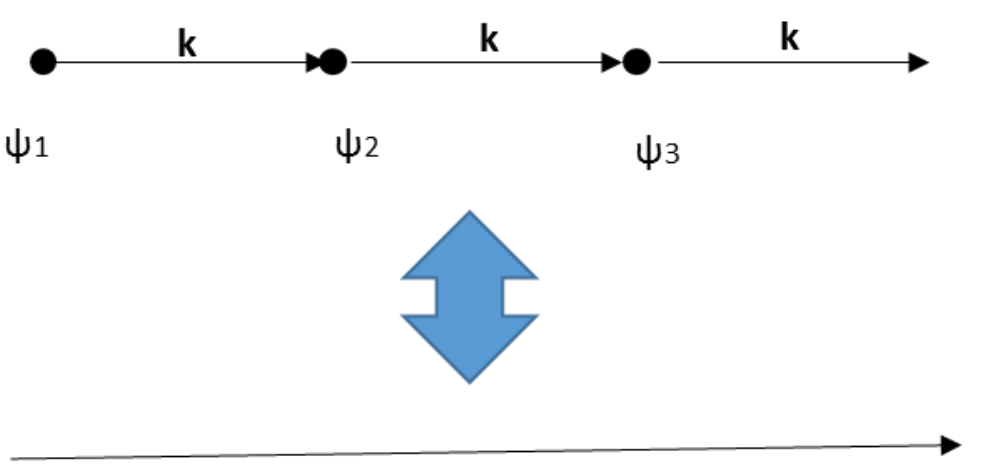

$3 \mathbf{k}$

Fig. 7

Schematic drawing showing the summation of each wave number along one dimension. In general, basic physics claims that a wave number in metal is a function of kinetic energy and thus must follow both Fermi statistics and the exclusion principle. However, in the present case, the wave number is a function of the electrostatic potential $\phi$, which allows each electron to possess an identical wave number. That is, each wave function has the same wave number as a part of the phase. Although a wave function takes each location, the summation of all one-dimensional wave number vectors is possible. Overall, it is suggested that each wave number can be added without contradicting the exclusion principle. That is, the upper figure is identical to the lower figure among Fig.7.

Considering a measurable electric potential, Equation (31) becomes substantially large. As will be reported in the Results (see Section 5), this substantially large electric potential is reproduced in the simulations. Finally, now we can obtain a macroscopic wave function:

$\psi_{M}=\left|\psi_{M}\right| \exp \left(-\frac{\mathrm{e} \Phi_{T}}{\mathrm{ch}} \mathrm{jx}\right)$,

$\int\left|\psi_{M}\right|^{2} d v=1$.

The macroscopic wave function suggests that a condensation occurs with fermions exclusively along one dimension under a specific condition.

\section{Method}

We use PSIM software to carry out numerical simulation, which is affordable. This software was developed to calculate many types of electric circuits. First, the results of our previous paper [1] are reviewed. In the next step, energy generation is obtained by employing a voltage-controlled current 
source.

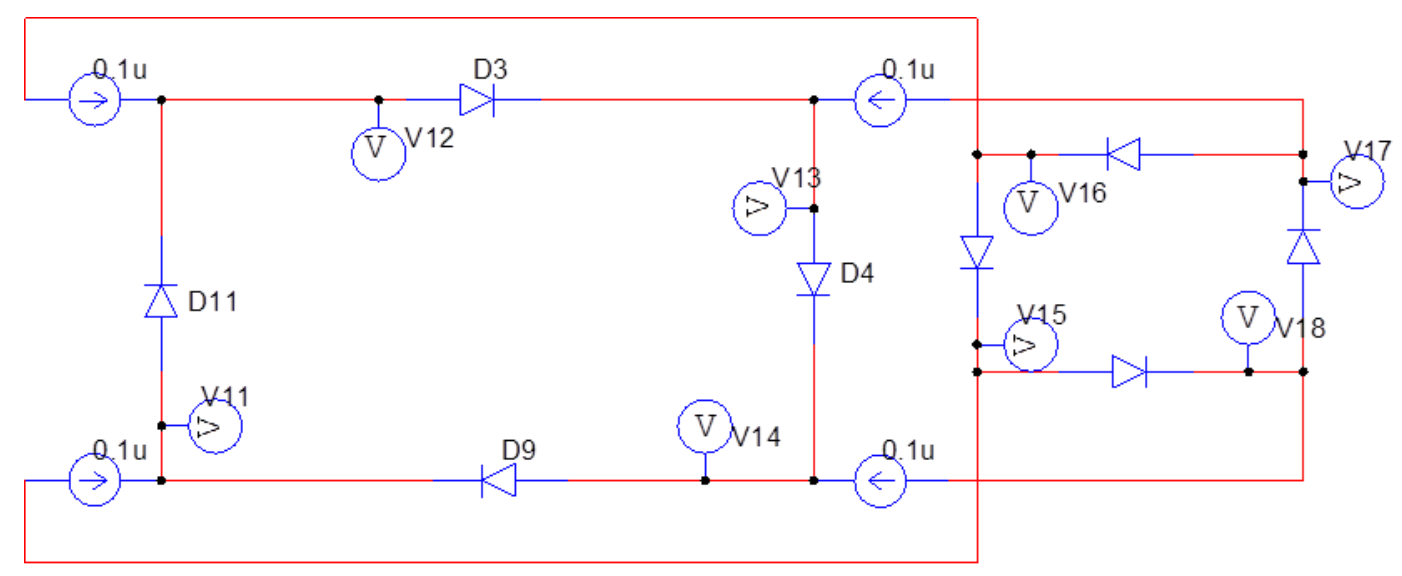

Fig. 8

Review circuit. The review of our previous work [1] is carried out to better understand the circuits developed in our current paper. Each independent current source inputs $0.1 \mu \mathrm{A}$. Positive electric potentials are measured by the probes V11-V14, and negative electric potentials are measured by the probes V15-V18.

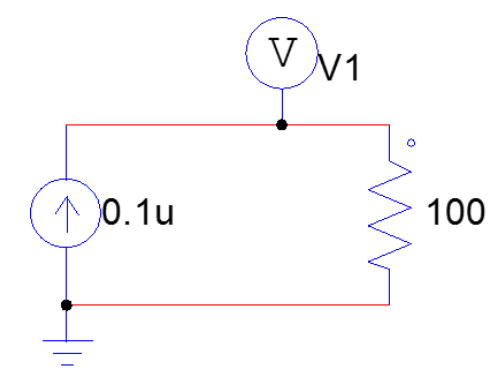

Fig. 9

An ordinary circuit for comparison with our proposed system circuit (Fig. 8). An input current from the current source is $0.1 \mu \mathrm{A}$, and the load is $100 \Omega$. The results regarding the probe V1 are covered in the Results (Section 5)

Fig. 8 shows the entire circuit including probes that was proposed in our previous paper [1]. As indicated in Result section, this system generates large electric potentials. Note that, the probes V11 to V14 will detect positive electric potential along the loop, and the probes V15 to V18 will detect negative electric potential along the other loop. Moreover, the initial input current from an independent current source is assumed to be $0.1 \mu \mathrm{A}$. For comparison and to understand the extent of the resulting electric potentials, Fig. 9 (i.e., a normal and ordinary electric circuit) was also simulated; the results are presented in Section 5. 
Fig. 10 shows the circuit when a voltage-controlled current source is added to the circuit shown in Fig. 8. As mentioned, the internal conductance $k$ is set to 1 . Note that the value of load $R_{L}$ is assumed to be $100 \mathrm{k} \Omega$. In the Results (Section 5), the output current $\mathrm{I}_{0}$ and input voltage $\mathrm{V}_{\text {in }}$ are calculated.

As described in Result section, the input current $I_{0}$ passing the load $\mathrm{R}_{\mathrm{L}}$ and the internal conductance $\mathrm{k}$ becomes very large. To protect the load $\mathrm{R}_{\mathrm{L}}$ and internal conductance $\mathrm{k}$ from Joule heating, a DC-DC conversion (i.e., rapid frequency switching) is further added to the input taps of the voltage-controlled current source, as shown in Fig. 11. Note that bi-directional switches are used in the PSIM simulation as a switching device, and switching frequency is assumed to be $10 \mathrm{kHz}$.

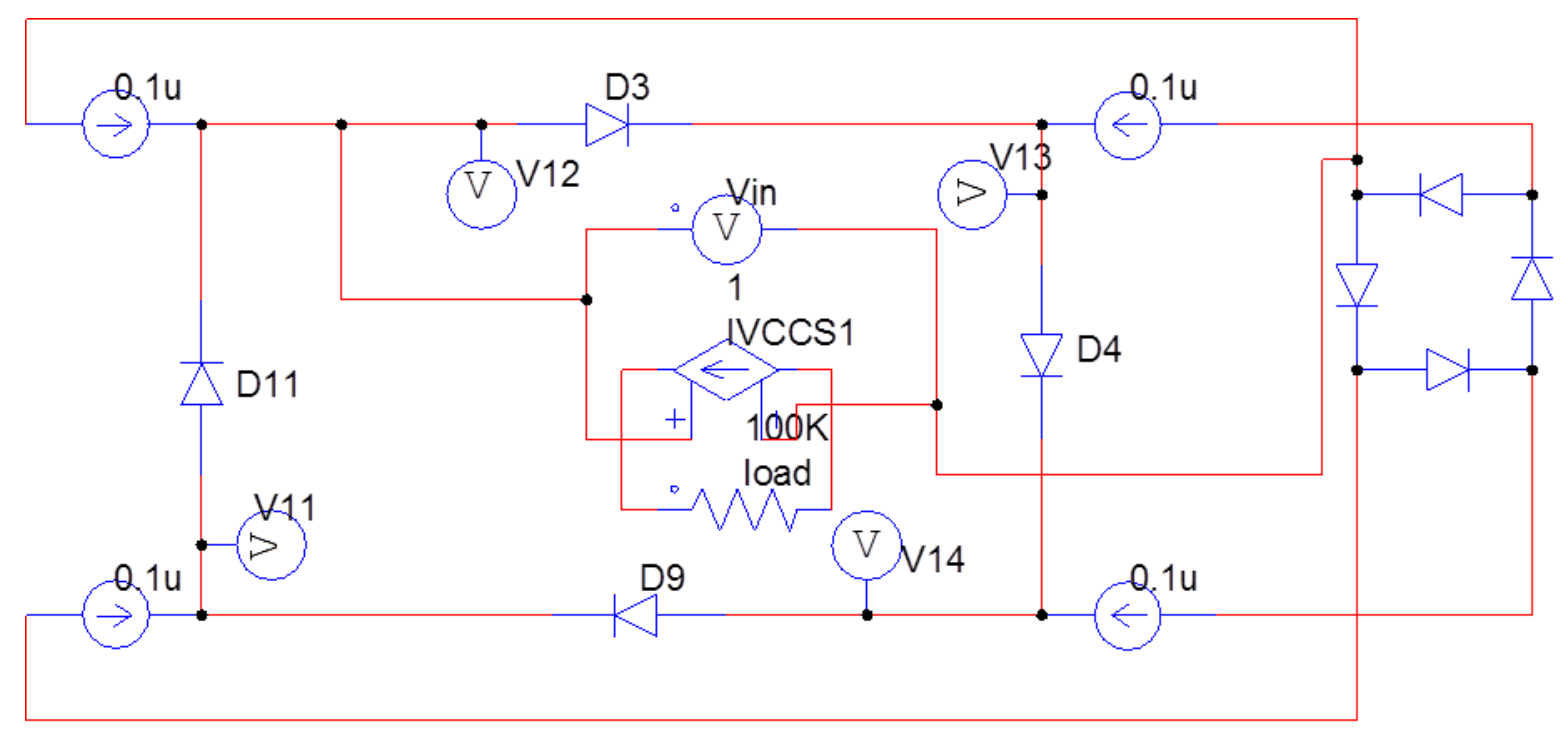

Fig. 10

The developed circuit, based on the original review circuit, is presented in Fig. 8. Here a voltage-controlled current source (named IVCCS1) is added. The input voltage $\mathrm{Vi}_{\mathbf{n}}$ is composed of both a loop undergoing positive electric potential as well as a loop undergoing negative electric potential. The load is assumed to be $100 \mathrm{k} \Omega$. 


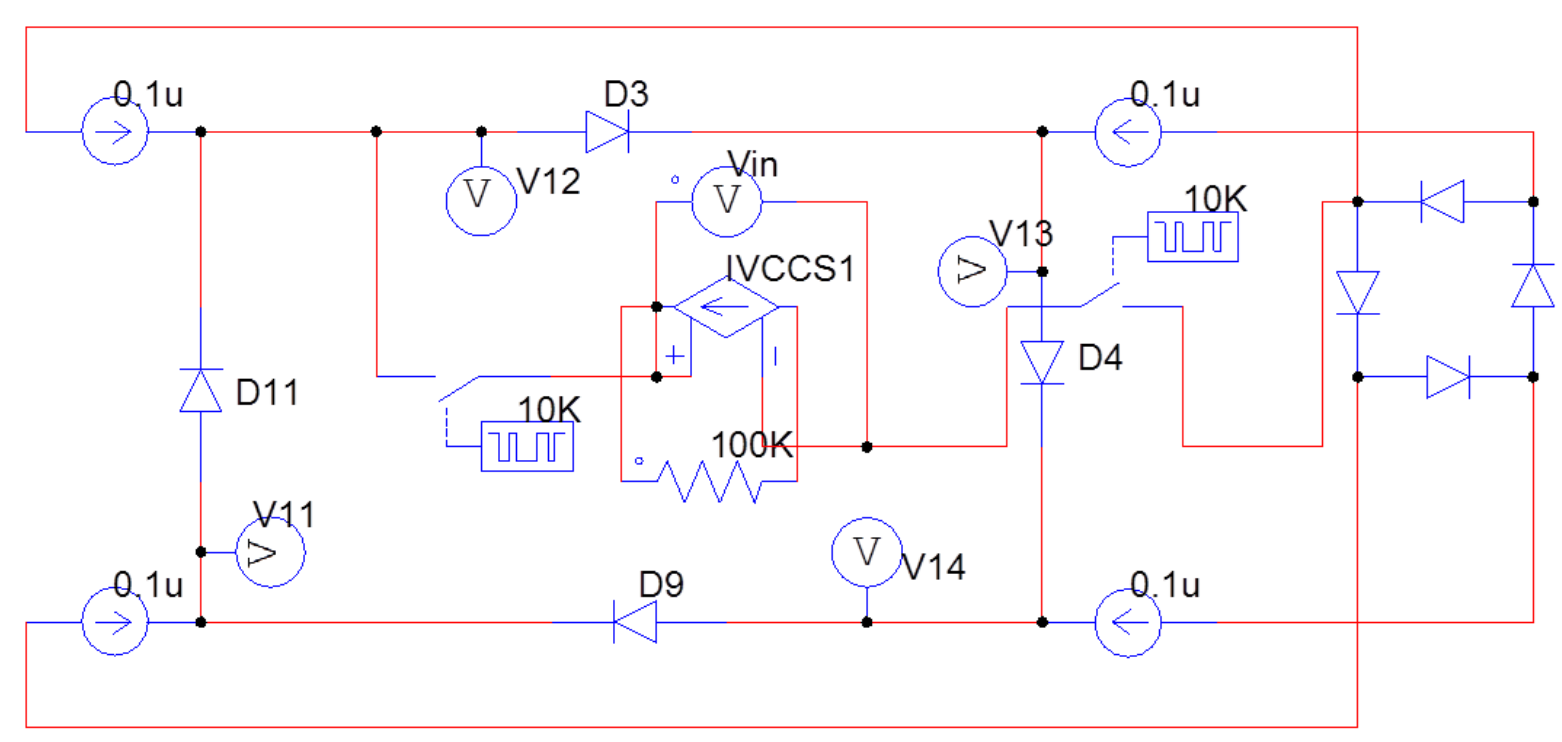

Fig.11

Schematic circuit diagram of the proposed system after bi-directional switches are introduced to the circuit presented in Fig. 10. To protect the internal conductance of the voltage-controlled current source from Joule heating, a DC-DC conversion is introduced. The switching frequency of the bi-directional switches is assumed to be $10 \mathrm{kHz}$. An increase in Joule heating, which increases linearly over time, is prevented. According to the PSIM simulation, the bi-directional switches undergo a current flow, which is calculated as an average, $2.2 \times$ $10^{-8}$ A.

\section{Result}

Simulation results of the circuits introduced in the Method section are presented.

In Fig. 12, the simulation results from the circuit shown in Fig. 8 are presented. Each loop has the same electric potential, i.e., no voltage along the loop, and one loop has a positively large electric potential, and the other loop has a negative large electric potential.

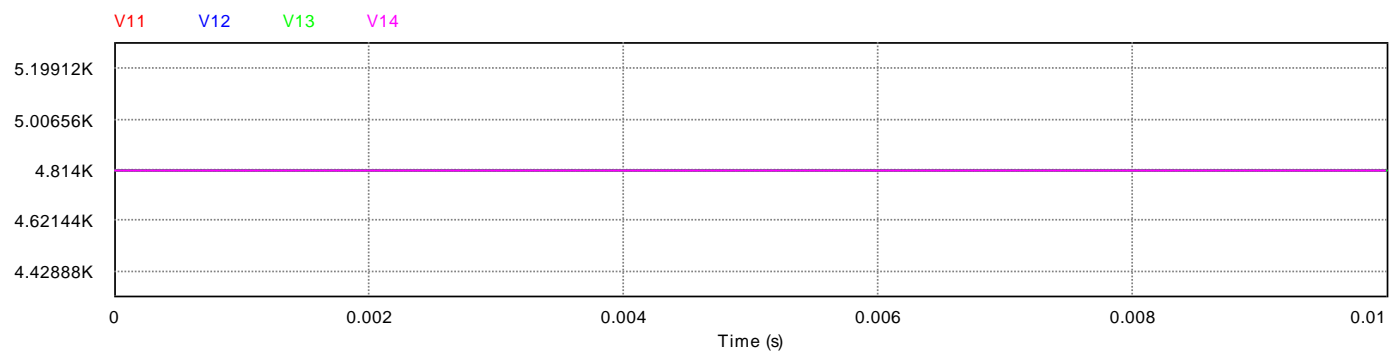




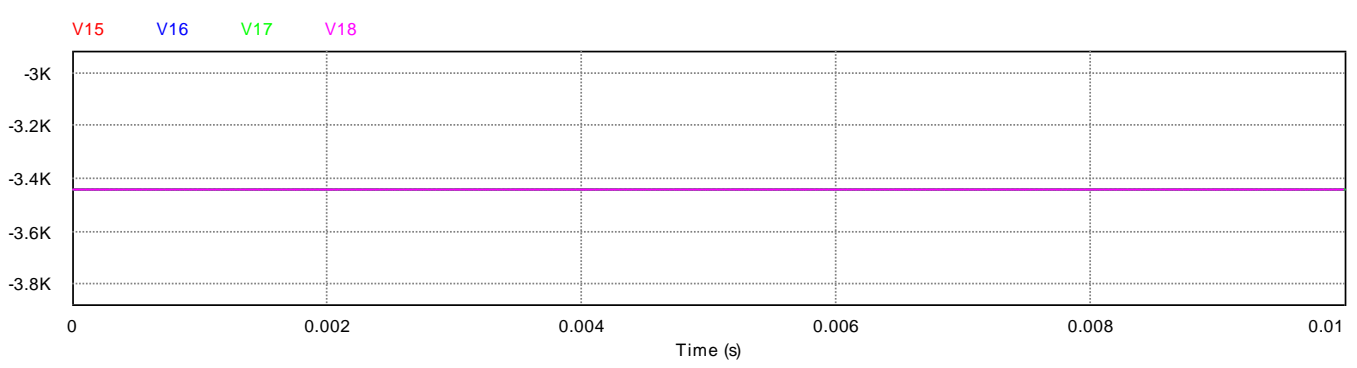

Fig.12

Simulation of review circuit in Fig. 8, using the PSIM software. Note that the unit of perpendicular axes is [V].The upper figure implies positive electric potentials with the 4 probes along the loop, whereas the lower figure indicates negative electric potentials with the 4 probes in the review circuit along the other loop. As shown, each loop has a uniform electric potential, which satisfies a condition of superconductivity.

A result of an ordinary circuit (from Fig. 9) is prepared for comparison in Fig. 13. As shown, normally $0.1 \mu \mathrm{A}$ input cannot generate large electric potential. Therefore, the proposed system involves new phenomena.

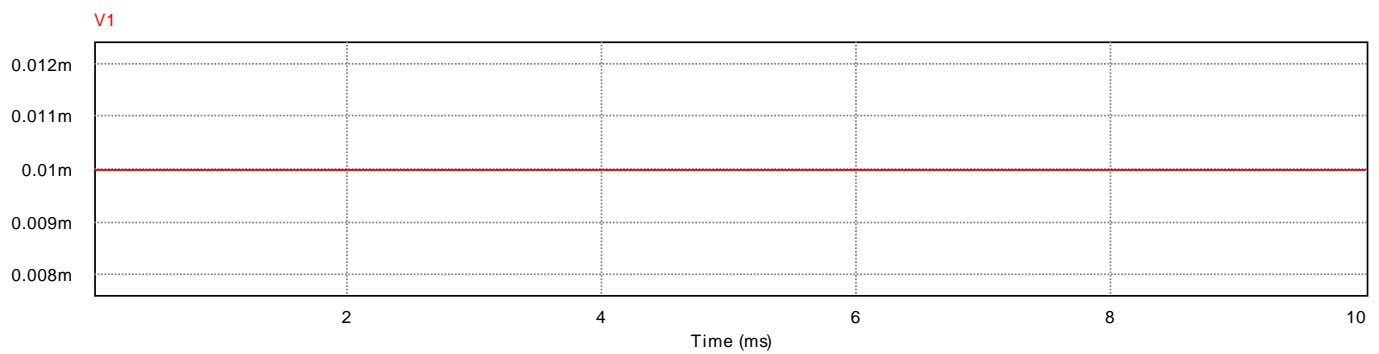

Fig. 13

The result of an ordinary circuit (shown in Fig. 9) in which an independent current source is used, and the load is $100 \Omega$. The unit of the perpendicular axis is [V]. Here, $0.1 \mu \mathrm{A}$ is transmitted to the $100 \Omega$ load. As shown, the resulting electric potential is small, $0.01 \mathrm{mV}$. In comparison, the proposed system generates $\mathrm{kV}$ order for $0.1 \mu \mathrm{A}$ initial input.

As shown in Fig. 14, the circuit (see Fig. 10) is simulated after adding a voltage-controlled current source. Here, $V_{\text {in }}$ refers to the input voltage to the voltage-controlled current source, and I(load) indicates the output current with the load $\mathrm{R}_{\mathrm{L}}$. As mentioned, because the internal conductance $\mathrm{k}$ is given the value 1 , a precise amount of the input voltage is converted to the output current. An important result is the successful generation of a very large net output current, i.e., large electric 
power.
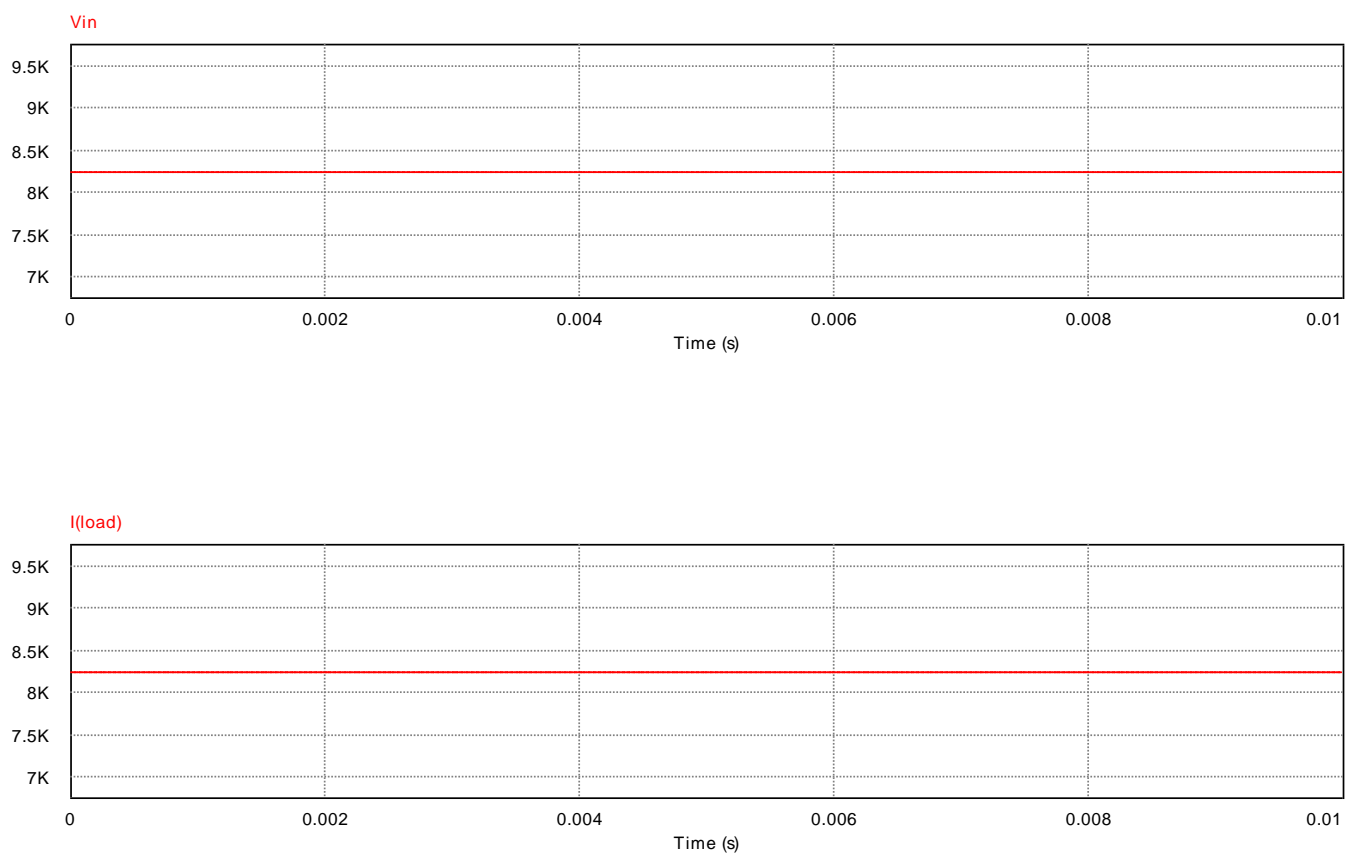

Fig. 14

Simulation of the circuit upon introducing a voltage-controlled current source (see Fig. 10). The upper figure indicates the input voltage $V_{\text {in }}$ (the unit is [V]). On the other hand, the lower figure implies the output current with the load (the unit is [A]). An extremely large output current is created because the voltage-controlled current source precisely converted the input voltage.

Figure 15 shows the simulated results upon introducing bi-directional switches to carry out DCDC conversion (from Fig. 11). By introducing this switching method, the Joule heating in the internal conductance $\mathrm{k}$ can be reduced. Note that in the process of this simulation, the PSIM software detected an extremely small current in the bi-directional switch. Due to this current, the input voltage $V_{\text {in }}$ is slightly reduced.

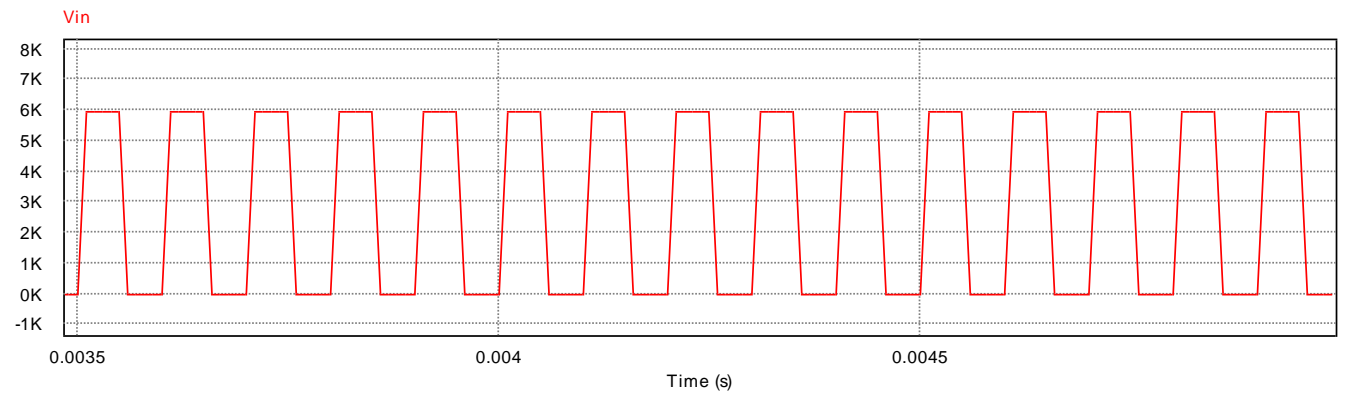




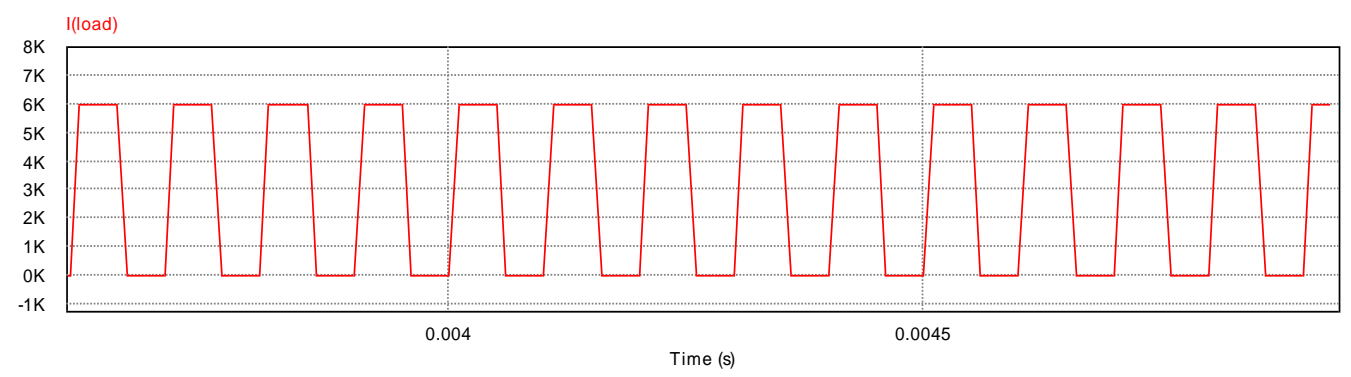

Fig. 15

Simulations of introducing bi-directional switches for a DC-DC conversion (see Fig. 11): the upper figure (the unit is [V]) is input voltage as a function of the voltage-controlled current source, and the lower figure (the unit is [A]) is output current over time. As mentioned, the bi-directional switches in the PSIM software undergo a real and very small current $\left(1^{-8} \mathrm{~A}\right)$ order which affects the value of the input voltage Vin, i.e., using bidirectional switches results in a slightly reduced input voltage $V_{\text {in. }}$

\section{Discussion}

\subsection{Theory}

This theory is based on Lorentz conservations described in Eqs (5)-(6). According to theses equations, states are categorized as transient and steady states. In a transient state, the right-hand sides of the Lorentz conservation equations are non-zero, and consuming the zero-point energy in these sides produces the steady state at which the vector and electrostatic potentials become much larger.

In the transient state, the existence of divergent current density was considered with the Dirac equation because the eigenvalue of this equation essentially corresponds to the zero-point energy on the right-hand side of the Lorentz conservation equation (Eq. 5). Using the normalizations of the wave function and the equation of continuity, the existence of divergent currents was derived at the connecting points of the independent current sources. This implies that the present system works correctly according to the theory, i.e., the currents are input to a loop from the independent current sources.

In the steady state, a London equation was obtained, which demonstrates the Meissner effect, by applying the two Lorentz conservation equations that hold in the steady state. Moreover, a summation of the wave numbers along the one-dimensional loop was proposed, thus forming a macroscopic wave function along the loop. These results suggest that the Meissner effect and macroscopic wave function coexist with the zero voltage along the loop despite the rather large electric potential. A feature of the proposed condensation from the macroscopic wave function is that it does not involve a combination 
of two electron pairs; therefore, this case does not involve a superconducting energy gap or a critical temperature. Accordingly, we suggest that this new type superconductivity does not require refrigeration and that a new type of condensation occurs.

\subsection{Simulations}

\subsubsection{Review}

As described in the Results (Section 5), the circuit in the review exhibits an identical electric potential at each point along the loop, which implies that a condition for superconductivity with a current and no voltage is satisfied. Moreover, the appearance of large electric potentials supports the presented theory.

\subsubsection{Magnitude of Load $R_{L}$}

An independent current source supplies a constant current to a load, but it has limitations due to its output impedance. That is, an independent current source cannot transmit a current to the load that is larger than its output impedance (i.e., average of about $10 \mathrm{M} \Omega$ ). In the present case, however, it is not necessary to consider such characteristics because a voltage-controlled current source is used. More concretely, consider Fig. 2, which depicts the structure of a voltage-controlled current source. Despite the output impedance and the load $\mathrm{R}_{\mathrm{L}}$ being connected in parallel, i.e., an output impedance between the inverting terminal and output tap of the op-amp is present, the current $I_{B}$ in Fig. 2 being nearly zero renders the existence of the parallel output impedance meaningless. This implies that output impedance need not be considered in a voltage-controlled current source. Therefore, a sufficiently large generating current is supplied despite the large load $\mathrm{R}_{\mathrm{L}}$. In the PSIM simulation, for a load for up to nearly100 $\mathrm{G} \Omega$, the generating output current remains constant as about $7 \mathrm{kA}$; however, for a higher load, the generating current quickly becomes nearly zero. Therefore, this study employed a load $\mathrm{R}_{\mathrm{L}}$ of $100 \mathrm{k} \Omega$, which is relatively large.

\subsubsection{Large Input Voltage and Input Impedance of an Op-amp}

If the large input voltage broke the high input impedance of the op-amp, it would imply that an Ohmic field-based current appeared from one loop to the other loop in the system. As mentioned, however, the input voltage is created from electrostatic fields, not Ohmic fields. That is, electrostatic potentials cannot determine a current according to Maxwell's equations. To conclude, there is no risk to inputting a large voltage derived from electrostatic potentials; we emphasize that this is allowed 
only in our system.

\subsubsection{DC-DC Converter}

This study employs a method based on a DC-DC converter, which is analogous to pulse-width modulation (PWM). The purpose of using this method stems from the output current from the voltagecontrolled current source being about $7 \mathrm{kA}$ and the fact that Joule heating in a resistance generally increases linearly with time; the DC-DC converter is necessary to secure the load $\mathrm{R}_{\mathrm{L}}$ and the internal conductance $\mathrm{k}$ from Joule heating. The DC-DC converter-based method used here is simply rapid interactions of on -off switching and enables the Joule heating to be constant and small (not increase with time). This method is common and is used worldwide, being taught in universities as power electronics.

Based on the results of the simulations, the average generating electric power $\mathrm{W}$ is

$$
\mathrm{W}=R_{L}<I>^{2},
$$

where $\langle\mathrm{I}\rangle$ denotes the time averaged output current that is arises from the DC-DC converter. Considering the average output current, the average generated electric power is calculated as

$$
\mathrm{W}=8.8 \times 10^{11}[\mathrm{~W}]
$$

Because a unit of electric power in ordinary thermal power stations is in the order of magnitude of $10^{9}$ $\mathrm{W}$, it is clear that the proposed simple system generates electric power much larger than in thermal power stations. If a load larger than that of Fig. 10 or Fig.11 (i.e., $100 \mathrm{k} \Omega$ ) is allowed, tan increased output of electric power will be generated. Considering that the initial input current from the 4 independent current sources is $4 \times 0.1 \mu \mathrm{A}$, and the average output current $\langle\mathrm{I}>$ is estimated to be 3.6 $\mathrm{kA}$; the gain of the current increase is $7.45 \times 10^{9}$ times.

Next, the Joule heating is estimated in the internal conductance $\mathrm{k}$ in the voltage-controlled current source. In the DC-DC converter, the constant Joule heating u is represented as

$\mathrm{u}=\left(\frac{1}{\mathrm{k}}\right) I_{0}^{2} T_{\text {on }}$,

where $\mathrm{I}_{0}$ and $\mathrm{T}_{\text {on }}$ denote the output current and time when the switch is on, respectively.

For a switching frequency of $10 \mathrm{kHz}$ and the duty-ratio in terms of the DC-DC conversion is $0.5, \mathrm{~T}_{\text {on }}$ is estimated as

$T_{\text {on }}=5.0 \times 10^{-5}[\mathrm{~s}]$.

Moreover, $\mathrm{I}_{0}$ is calculated by PSIM software as

$I_{0}=5.9 \times 10^{3}[\mathrm{~A}]$.

Considering that $1 / \mathrm{k}$ is defined as $1 \Omega$, the Joule heating in the internal conductance $\mathrm{k}$ is

$\mathrm{u} \approx 1.7 \times 10^{3}[\mathrm{~J}]$.

This value is considered relatively small despite the large output current. Generally, kJ heating is generated when making a chemical compound. In our opinion, it is therefore not necessary to cool the 
conductance k with water. For instance, when $1 \mathrm{~mol}$ of $\mathrm{CH}_{4}$ is burned, $891 \mathrm{~kJ}$ heating is generated [19]. In comparison, the calculated Joule heating in this system, u, is much smaller. However, we suggest consulting electronics experts to ascertain if water cooling is necessary.

\subsection{Contribution}

\subsubsection{Contribution to Condensed Matter Physics}

The first contribution of this study is that we show the existence of divergent current density, considering the Lorentz conservation (i.e., the zero-point energy in the conservation energy) and Dirac equation, which has an eigenvalue based on the two zero-point energies. Such an indication of divergent current density has not been reported thus far. This is significant in the relativity, electromagnetic field theory, and basic quantum mechanics.

Secondly, it is significant that this work describes a new superconductivity that is independent of temperature (i.e., superconductivity with no critical temperature). The history of superconductivity suggests that enhancements of the critical temperature are necessary to use superconductors at room temperature. Recently, it was reported that very high pressures provide high critical temperature near room temperature [14]. Although such results are important in physics, technical merits and actual application have not been reported. In comparison, our circuit-based approach, which has not been reported elsewhere readily results in the new and temperature-independent superconductivity reported here, which is also described in [15-18]. The major difference between our previous reports on new superconductivity and the present paper is that the superconductivity reported herein does not require the formation of electron pairs. It therefore simultaneously implies that the reported superconductivity has no superconducting energy gap or critical temperature. Each wave number is a function of an electric potential, not a kinetic energy, and thus, it is allowed that each electron possesses an identical wave number. Such a one-dimensional wave number for each wave function enables their summation as vectors. This does not contradict the exclusion principle, as described with Fig. 7; instead, it results in the formation of a macroscopic wave function and condensation. This type of condensation is not related to local thermal equilibriums, thus basic quantum statistics in thermal equilibrium such as Fermi statistics are not applied. We claim that this fermion-based condensation can be formed only under a specific condition, which has not been fundamentally proposed but would result deeper understanding in condensed matter physics.

\subsubsection{Technological Contribution}

In our previous study [1], we demonstrated that uniform and a large electric potential appears along 
a loop; therefore, a condition of superconductivity is satisfied. However, the uniform electrostatic potential distribution along a loop implies there is no net voltage; hence, energy generated from voltage could not be obtained in the previous paper.

To solve this problem, a voltage-controlled current source is introduced in the present paper. Moreover, we found in the present paper that among two superconducting loops in the system, one has a positive electrostatic potential, whereas the other has a negative electrostatic potential. This fact is used to input a large DC voltage to the voltage-controlled current source. As a result, a DC current on the order of several kA was obtained despite the initial input current from the independent current source being less than $1 \mu \mathrm{A}$. If the load of the voltage-controlled current source is sufficiently large, the simulation reveals that energy much larger than that of thermal power station can be generated. Compared with thermal power stations, our system generates little heat loss, making the energy conversion ratio very efficient.

Moreover, the energy density in the present system is very high. Although the initial input current is less than $1 \mu \mathrm{A}$, the resulting current and energy dominate those of a unit of energy generated in thermal power stations. This implies that investing in plant and equipment is not necessary with the proposed system, making is available to average and private laboratories.

The proposed system is very inexpensive to install. Because the initial input current from the independent current source is very small, the costs of the independent current sources are expected to be less than 100,000 yen (1,000 USD). Concerning a voltage-controlled current source, the internal structure of the circuit in Fig. 2 is simple, using an op-amp in it; in short, this technology is accessible and could be installed by a student. Low cost is still expected if the voltage-controlled current source is purchased.

Furthermore, the proposed energy generating system creates no atomic radiation and requires no fossil fuels.

To conclude, if our system is put into practice, the results presented herein imply that energy can be generated inexhaustibly in a net sense and that trading energy for financial compensation will be meaningless. If successful, dependency on fossil fuel-rich regions of the world (Middle East and Russia) will be significantly reduced or removed. The current economic system, in which a few people are involved in profiting from energy and hence possess the vast majority of the worldwide wealth, will collapse, thus resulting in reducing the gap between the rich and the poor.

\section{Conclusion}

We proposed a new energy generation system and analyzed it theoretically and numerically. The results clarified that very large energy density can be obtained from the system at minimal cost and with no radiation or fossil fuel consumption. The gained energy density is much larger than that the 
average unit of energy from a thermal power station. Therefore, we believe that this system will be a candidate for a complete replacement of the current fuel-dependent energy generation stations. As a follow-up, we plan to confirm the results further with experiments.

\section{References}

[1] S. Ishiguri, New Systematic Energy Generation Having Superconductivity Properties without Refrigeration, Preprints 2019070082 (doi:10.20944/preprints201907.0082.v1). (2019)

[2] R. Williams, The Journal of Chemical Physics, 32 (5), 1505-1514, (1960)

[3] M. Gotz, et al, Renewable Energy, 85, 1371-1390 (2016)

[4] J. Pitel and P. Kovac, Supercond. Sci. Technol 10847 (1997)

[5] P. Benioff, Journal of Statistic Physics, 22, 563-591 (1980)

[6] P. W. Anderson, et al, J. Phys.: Condens. Matter, 16, No. 24, R755 (2004)

[7] F. C. Zhang, T. M. Rice, Phys. Rev., B 37, 3759 (1988)

[8] M. Ogata, H. Fukuyama, Rep. Prog. Phys., 71, (2008)

[9] J. G. Bednorz and K. A. Müller, Zeitschrift für Physik, B 64, 189 (1986)

[10] J. Nagamatsu, et al, Nature, 410, 63 (2001)

[11] Y. Kamihara, et al, J. Am. Chem. Soc, 128 (31), 10012-10013 (2006)

[12] J. Bardeen, L. Cooper, J.R. Schrieffer, Phys. Rev., 108, 1175 (1957)

[13] B.D.Josephson, Physics Letters, 1 (7), 251-253 (1962)

[14] M. Somayazulu, et al, Phys. Rev. Lett. 122, 027001 (2019)

[15] S. Ishiguri, J. Supercond. Nov. Magn. 24, 455 (2011)

[16] S. Ishiguri, Int. J. of Mod. Phys. B, 27, 1350045 (2013)

[17] S. Ishiguri, "New Superconductivity and Theoretical Study on a New Phenomenon of Energy Source with Assistance of Initial Experiments." Preprints, 2018110636 (doi: 10.20944/preprints201811.0636.v1). (2019)

[18] S. Ishiguri, "Theory on Another Type of Temperature-Independent Superconductivity Based on Circuit Approaches with High Critical Current Density,” Preprints, 2019110033 (2019)

[19] M. Shimoi and S. Murata, Introduction of Chemistry $2^{\text {nd }}$ version, p.80, Tokyo-Kagaku-Dojin in Tokyo (2016)

\section{Additional information}

This paper is not related to any competing interests such as funding, employment and personal financial interests. Moreover, this paper is not related to non-financial competing interesting. 


\section{Acknowledge}

We thank Enago (www.enago.jp) for English language Review. 